\title{
Effect of Carbonate Apatite Hydrogel-Advanced Platelet-Rich Fibrin Injection on Osteoblastogenesis during Orthodontic Relapse in Rabbits
}

\author{
Ananto Ali Alhasyimi $i^{1, \odot}$ Sri Suparwitri ${ }^{1, \odot}$ Christnawati Christnawati \\ ${ }^{1}$ Department of Orthodontics, Faculty of Dentistry, Universitas \\ Address for correspondence Sri Suparwitri, Department of \\ Gadjah Mada, Yogyakarta, Indonesia \\ Orthodontics, Faculty of Dentistry, Universitas Gadjah Mada, \\ Yogyakarta 55281, Indonesia (e-mail: suparwitri_fkg@ugm.ac.id).
}

Eur J Dent 2021;15:412-419

\begin{abstract}
Objective This study aimed to determine the effect of carbonate apatite (CHA) hydrogel-aPRF on osteoblastogenesis during relapse in rabbits.

Materials and Methods Forty-five rabbits were divided into three groups $(n=15)$ : the control, CHA, and CHA-autologous platelet-rich fibrin (aPRF) groups. An open-coil spring was compressed between brackets to distalize the lower incisors of the rabbits by delivering a force of $50 \mathrm{cN}$ for 1 week. The new position of the teeth was retained for 14 days, and CHA hydrogel-aPRF was injected every 7 days. The appliances were then debonded to allow relapse. On days $0,3,7,14$, and 21 after debonding, transforming growth factor (TGF)- $\beta 1$ and bone morphogenetic protein (BMP)- 2 expression was examined using immunohistochemistry staining and Runx-2 levels were analyzed by enzyme-linked immunosorbent assay. The data collected were analyzed using analysis of variance and a post hoc Tukey's test $(p<0.05)$.

Results Histomorphometric analysis revealed that TGF- $\beta 1$ expression in the CHA-aPRF group is statistically higher than that in other groups on days 0,3 , and 7 after debonding $(p<0.05)$. BMP-2 expression in the CHA-aPRF group was also statistically higher than that

Keywords

- orthodontic relapse

- carbonate apatite

- platelet-rich fibrin

- osteoblastogenesis

in the other groups on days 3, 14, and 21 after debonding $(p<0.05)$. ELISA showed that Runx-2 levels are slightly higher in the CHA-aPRF group than in the other groups $(p>0.05)$. Conclusion Although injection of CHA-aPRF aids in osteoblastogenesis associated with enhancing TGF- $\beta 1$ and BMP-2 expressions, it does not significantly upregulate Runx-2 levels.
\end{abstract}

\section{Introduction}

Orthodontic relapse, which can be defined as a tendency of the teeth to return to their former positions after tooth movement, is an undesirable outcome of orthodontic treatment. ${ }^{1}$ A previous study reported relapse postorthodontic treatment rates of approximately 70 to $90 \%{ }^{2}$ Relapse recognized as a major challenging orthodontic clinical issue. A more comprehensive understanding of the relapse process is needed to determine ways to alleviate relapse or reinforce retention. Considering

published online

December 26, 2020
DOI https://doi.org/

10.1055/s-0040-1721234 ISSN 1305-7456. the complexity of relapse movement, its etiology remains unclear. An animal study revealed that osteoclastogenesis performs an important role in relapse. ${ }^{3}$ Osteoblastogenesis is believed to counteract the osteoclastogenesis. ${ }^{4}$ Osteoblasts, the principal mononucleated cells of bone tissue, are responsible for alveolar bone formation and the stability of the moved tooth. ${ }^{1}$ Transforming growth factor- $\beta 1$ (TGF- $\beta 1$ ) and bone morphogenic protein-2 (BMP-2) expression plays fundamental roles in osteoblastogenesis. Mitogen-activated protein kinase signal activates by TGF- $\beta 1$ and BMP-2 positively regulate Runt-related

(C) 2020. European Journal of Dentistry.

This is an open access article published by Thieme under the terms of the Creative Commons Attribution-NonDerivative-NonCommercial-License, permitting copying and reproduction so long as the original work is given appropriate credit. Contents may not be used for commercial purposes, or adapted, remixed, transformed or built upon. (https://creativecommons.org/licenses/by-nc-nd/4.0/)

Thieme Medical and Scientific Publishers Pvt. Ltd., A-12, 2nd Floor, Sector 2, Noida-201301 UP, India 
transcription factor-2 (Runx-2) expression and promotes mesenchymal stem cell (MSC) differentiation. ${ }^{5}$ Runx-2, also known as core-binding factor subunit $\alpha-1$, is a transcription factor that is essential for differentiating MSCs into osteoblasts. ${ }^{6}$

In general, the prevention of orthodontic relapse is attempted mechanically using a retainer. Fixed retainers are most regularly used in the orthodontic retention phase as they have several benefits, such as low patient cooperation requirements, good effectiveness, pleasing aesthetics, and suitability for lifelong retention. However, their need for precise bonding techniques and their tendency to induce negative effects on periodontal health limit their uses. ${ }^{7}$ Besides, relapse could may still occur even after 10 years of retainer usage. ${ }^{8}$ Orthodontic relapse prevention is also ameliorated pharmacologically by drugs such as bisphosphonates. Unfortunately, bisphosphonates induce unwanted effects on patients, including an oral intolerance, and jaw bone osteonecrosis. ${ }^{9}$

Up to now, tissue engineering approaches are also advocated to manipulate alveolar bone remodeling, blocking orthodontic relapse, and enhancing the stability of tooth positions as well. ${ }^{1,10}$ Carbonate apatite (CHA) exhibits good potential for bone tissue engineering because of its well-controlled calcium release and bone formation ability. ${ }^{11} \mathrm{CHA}$ is considered an excellent biomaterial for improving alveolar bone remodeling on account of the similarity of its structure to the interconnected porous structure of bone. ${ }^{12} \mathrm{CHA}$ has also received increased attention for its ability to act as a drug delivery system for protein delivery into living cells. ${ }^{13}$

The development of autologous platelet-rich fibrin (PRF), which can be produced via a minimally invasive procedure, could fulfill the demand for clinically applicable cell-based tissue engineering strategies in the context of bone cells prevascularization. PRF possesses excellent biological capability as a source of proteins vital to bone regeneration, including TGF- $\beta$, insulin-like growth factor (IGF), and platelet-derived growth factor (PDGF). ${ }^{14}$ Chang et al ${ }^{15}$ proved that PRF can stimulate significant osteoprotegerin (OPG) expression in vitro. Increases in OPG expression level indicate the enhancement of differentiated osteoblasts and is related to lower relapse rates. ${ }^{3}$ Advanced-PRF (aPRF) is a novel PRF modification form prepared by reducing the centrifugation speed and extending the centrifugation time of the original fibrin. This adjustment yields higher platelet concentrations, which is the result of fewer cells moving to the bottom of the tubes during centrifugation, and a greater amount of proteins, including platelets, left in the higher part of the tubes where the clot is isolated. ${ }^{16}$ The present study was designed to examine the potential of intrasulcular injection of CHA-aPRF to induce osteoblastogenesis during experimental orthodontic relapse movement in rabbits. The hypothesis of this study was that CHA-aPRF induce osteoblastogenesis by enhancing TGF$\beta 1$, BMP-2 expressions, and Runx-2 levels during orthodontic relapse in rabbits.

\section{Materials and Methods}

\section{Experimental Animals}

All animal experimental methods were conducted in accordance with the guidelines of the National Institutes of Health
Guide for the Care and Use of Laboratory Animals, and ethical clearance was obtained from the Research Ethics Committee of the Faculty of Dentistry. We obtained 10- to 12-week-old rabbits (Oryctolagus cuniculus) weighing approximately 2.5 to $3 \mathrm{~kg}$ from a commercial supplier. Male rabbits were selected because they are unaffected by fluctuations in estrogen. The rabbits were anesthetized in each experiment. The experiments began with a 1-week acclimatization to adapt the rabbits to their new environment and laboratory food. All of the rabbits were maintained individually in polycarbonate cages $\left(0.90 \times 0.60 \times 0.60 \mathrm{~m}^{3}\right)$ with an alternating light/ dark (12 hours/12 hours) cycle and standard temperature $\left(23 \pm 2^{\circ} \mathrm{C}\right)$ and humidity $(55 \pm 5 \%)$. A pellet diet (expanded pellets; Stepfield, United Kingdom) and tap water were fed to the rabbits ad libitum to minimize the possibility of bracket detachment and prevent eating difficulty during orthodontic tooth movement (OTM). A total of 45 rabbits were randomly divided into three groups $(n=15)$ : G1 group (i.e., those did not receive any injection), G2 group (i.e., those received intrasulcular injection of CHA hydrogel), and G3 group (i.e., those that received intrasulcular injection of CHA hydrogel-aPRF). Each group was randomly divided into five subgroups $(n=3)$ according to the observation day.

\section{Material Preparation}

CHA hydrogel was prepared by mixing type- $\beta$ gelatin, sodium citrate, and distilled water. This mixture was then added with calcium hydroxide and stirred using a magnetic stirrer for 1 hour. Phosphoric acid was dissolved in $50 \mathrm{~mL}$ of distilled water and then gently dropped into the gelatin mixture. The specimen was subsequently pulverized and filtered through a 32- $\mu \mathrm{m}$ mesh. Preparation of aPRF began with the anesthetization of the rabbits in the G3 group by intramuscular injection of $35 \mathrm{mg} / \mathrm{kg}$ body weight (BW) ketamine and $5 \mathrm{mg} / \mathrm{kg}$ BW xylazine. Approximately $10 \mathrm{~mL}$ of the rabbits' blood was collected using a hematocrit syringe through the marginal vein in the ear. The fresh blood was immediately transferred to a tube without anticoagulants and centrifuged at 1,500 rpm for 14 minutes. The middle layer of aPRF $(0.6-0.8 \mathrm{~mL})$ was separated from the two layers using tweezers and sterile scissors or punch biopsy and then compressed using a PRF box for 30 minutes to release the releasate.

Immediately after preparation, $200 \mu \mathrm{L}$ of the aPRF releasate was dripped onto $10 \mathrm{mg}$ of CHA that had been sterilized in an autoclave at $105^{\circ} \mathrm{C}$ for 60 minutes and incubated for 1 hour at $37^{\circ} \mathrm{C}$ to allow electrostatic binding between the releasate and hydrogel.

\section{Orthodontic Tooth Movement and Relapse Movement}

The rabbits were anesthetized by intramuscular injection with a concoction of ketamine ( $35 \mathrm{mg} / \mathrm{kg} \mathrm{BW}$ ) and xylazine $(5 \mathrm{mg} / \mathrm{kg} \mathrm{BW}$ ). An elastic separator was then placed between their two incisors to facilitate bracket installation. The labial surface of the lower incisors was cleaned with a brush, and straight wire brackets were bonded on to the teeth with a $0.016 " \times 0.016$ " stainless steel square wire inserted into the bracket slots. A Ni-Ti 0.010 " $\times 0.030$ " open-coil spring (DynaFlex; Missouri, United States) was attached between 
the two brackets to deliver a distal orthodontic reciprocal force of $50 \mathrm{cN}$. Installation of power $\mathrm{O}$ was performed to tie the wire attached to the bracket. The length of the stainless steel wire was $4 \mathrm{~mm}$ longer than the length of the opencoil spring to provide tolerance if OTM occurred. The end of the wire was bent to prevent mucosal wounding or injury (-Fig. 1).

An interincisal distance of $\pm 3 \mathrm{~mm}$ (measured from the mesial tips of the lower incisors) was obtained after the tooth had moved for approximately 7 days, and the open-coil spring was no longer active. At the end of active tooth movement, a rectangular stainless steel wire with a diameter of 0.016 " $\times$ 0.022 " was installed to replace the previous wire. The opencoil spring was not removed from the bracket slot to serve as a retainer. A distance of $\pm 3 \mathrm{~mm}$ was maintained for 14 days as a stabilization (retention) period. The wire, bracket, and opencoil spring were removed after the stabilization period, and the lower incisors were allowed to start relapse movement. The timeline in $\mathbf{- F i g}$. 2 shows the experimental design for the establishment of orthodontic retention in a rabbit model, including the time of active OTM, space retainment using a passive open-coil spring, and orthodontic relapse movement.
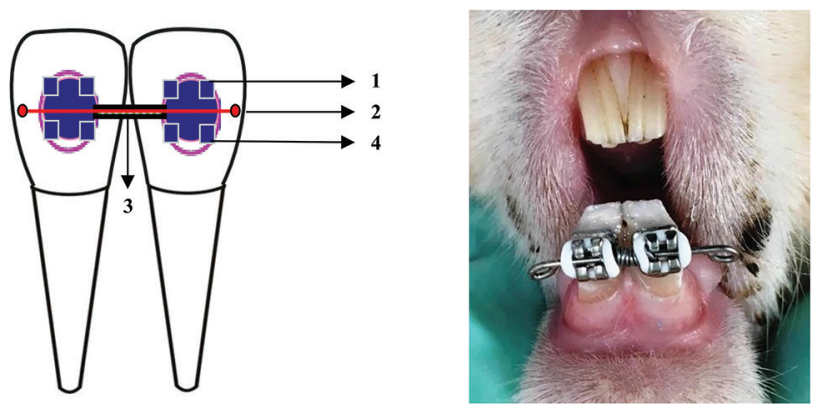

Fig. 1 Schematic of the experimental rabbit OTM model. (1) Straight-wire lower-incisor bracket slot, 0.022." (2) Stainless steel square wire, $0.016 " \times 0.016$." (3) nickel-titanium open-coil spring, 0.010 " $\times 0.030$." (4) Elastic power O. The length of the wire was $4 \mathrm{~mm}$ longer than the length of the open-coil spring to provide tolerance if OTM occurred. The end of the wire was bent to prevent mucosal wounding or injury. OTM, orthodontic tooth movement.

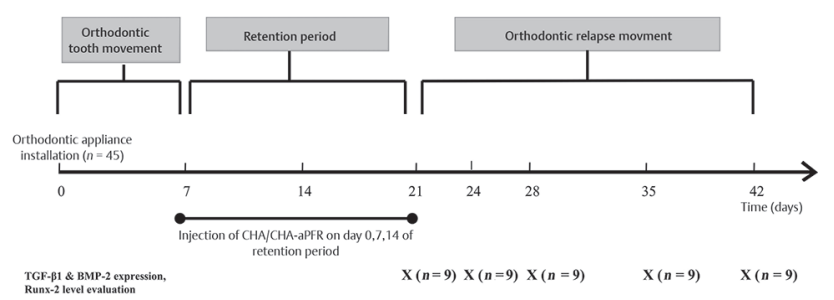

Fig. 2 Experimental timeline for the establishment of the rabbit relapse movement model. Rabbits were randomly divided into three groups: control (G1), CHA hydrogel injection (G2), and CHA hydrogel-aPRF injection (G3). Each group included 15 rabbits, and all rabbits received an orthodontic force of $50 \mathrm{cN}$ for 7 days, following 14 days of retention period. Rabbits in $\mathrm{G} 2$ and $\mathrm{G} 3$ received $\mathrm{CHA}$ and $\mathrm{CHA}-\mathrm{aPRF}$ injection every 7 days during the retention period. The period of orthodontic relapse began after appliance debonding for all groups. Three rabbits were sacrificed on five subsequent time points $(0,3,7,14$, and 21 days after debonding) in each group. aPRF, autologous platelet-rich fibrin; CHA, carbonate apatite.
Intrasulcular injection of $0.2 \mathrm{~mL}$ of $\mathrm{CHA}$ and $\mathrm{CHA}$ hydrogel-aPRF into the mesial side of the gingival sulcus of the incisor was administered three times to the treatment groups on days 0,7 , and 14 of the stabilization period under general anesthesia. This protocol complied with the rule that blood should be collected from experimental animals at 7-day intervals to avoid anemia.

\section{Histological Preparation and Analysis}

Specimens of rabbit mandibular tissue were retrieved on days $0,3,7,14$, and 21 postdebonding. The rabbits were euthanized with an overdose of anesthesia (lethal dose: $250 \mathrm{mg} / \mathrm{kg}$ BW ketamine and $50 \mathrm{mg} / \mathrm{kg}$ BW xylazine) and decapitated, and their lower alveolar bone was dissected. The dissected tissue was fixed with $10 \%$ formalin buffer solution for 24 hours in a tissue pot. The sample was subsequently demineralized using $10 \%$ ethylene diamine tetraacetate (pH 7.4) at room temperature for \pm 8 weeks. Each specimen was embedded in paraffin wax and vertically sectioned into $4 \mu \mathrm{m}$-thick serial slices at $50 \mu \mathrm{m}$ intervals. Histological analysis was conducted using immunohistochemistry staining.

Staining began with deparaffinization with xylol followed by rehydration in a graded ethanol series. The specimen was immersed in $3 \% \mathrm{H}_{2} \mathrm{O}_{2}$ in methanol for 30 minutes in a staining jar. Antigen retrieval was performed enzymatically using $0.1 \%$ proteinase $\mathrm{K}$ in PBS $(\mathrm{pH} 7.4)$ for 20 minutes. The object-glass was placed inside a humid chamber, dripped with sniper background solution (Starr Trek, BioCare, United States), and then left to stand for 10 minutes. TGF- $\beta 1$ and BMP-2 antibody solutions (Abcam, Cambridge, United Kingdom) were diluted with $1 \%$ bovine serum albumin in phosphate buffer saline solution (PBS) (pH 7.4) at a ratio of 1:100 for overnight culture (16 hours) at $4^{\circ} \mathrm{C}$. The antibody solutions were then removed, and the beaker was placed in a staining jar containing PBS (pH 7.4) and shaken for 5 minutes. The filled glass-object was then dropped with avidin-HRP label track and incubated for 10 minutes.

Next, the glass object was applied with a chromogen diaminobenzidine solution, dried, and applied with Meyers hematoxylin counterstain. Rehydration through a graded alcohol series followed. Clearing with xylol was subsequently performed to give a clear color to the tissues, and mounting with DPX-Mountant was performed to improve the durability and clarity of the samples.

Determination of TGF- $\beta 1$ and BMP-2 expression was performed by calculating the number of osteoblasts, which was positively expressing TGF- $\beta 1$ and BMP-2 in five levels derived from the root area of incisors mesial aspect: 100 , $140,180,220$, and $260 \mu \mathrm{m}$ (region of interest [ROIs]), with a $\times 400$ magnification light microscope and equipped with Optilab connected to ImageJ (NIH, Maryland, United States). Brown osteoblast's cytoplasm or nuclei was considered as positive cells. The number of TGF- $\beta 1$ and BMP-2 expression was obtained by calculating the average of positive cells across the five ROIs. All measurements were performed by two trained investigators who were blinded to the samples under study. 


\section{Runx-2 Levels Analysis}

Following gingival crevicular fluid (GCF) sample collection, the gingival sulcus of each rabbit was gently air dried. GCF samples were collected from all groups on days $0,3,7,14$, and 21 alternately during relapse movement using a 20 paper point. The paper point was gently inserted approximately $1 \mathrm{~mm}$ into the mesial side of the gingival sulcus of the incisor and then left in situ for 60 seconds; collection was performed twice. Two dipped paper points were then placed in a $1.5-\mathrm{mL}$ Eppendorf tube containing $350 \mu \mathrm{L}$ of physiological saline solution. Afterward, the tube was centrifuged at 2,000 rpm and $4^{\circ} \mathrm{C}$ for 5 minutes.

Enzyme-linked immunosorbent assay (ELISA) was conducted to assess Runx-2 levels during relapse movement. Analysis was conducted using a quantitative antiRunx-2 antibody-specific ELISA kit (ab76956, Abcam). The total amount of the transcription factor was compared with its standard curve. The optical density of the solutions was determined at $450 \mathrm{~nm}$ using a microplate reader, and the total level of Runx-2 was represented as picograms per milliliter $(\mathrm{pg} / \mathrm{mL})$.

\section{Statistical Analysis}

The data obtained in this study were statistically analyzed using two-way analysis of variance to determine differences and interactions between groups. Tukey's honest significant difference test was then performed to determine significant differences between groups. A $p$-value $<0.05$ was considered statistically significant. Analyses were performed using the Statistical Package for the Social Sciences version 21 (IBM, United States).

\section{Results}

The animals tolerated all experimental procedures well. No observable effects on overall health and no visible side effects of CHA and CHA-aPRF injection on the periodontal tissue of the rabbits were noted. The means and standard deviations of the total TGF- $\beta 1$ and BMP- 2 expression and Runx-2 levels of the three groups are summarized in - Table 1. Shapiro-Wilk normality tests for TGF- $\beta 1$ expression, BMP-2 expression, and Runx-2 level confirmed our assumption of normality.

In general, the numbers of TGF- $\beta 1$ and BMP-2-positive cells in the $\mathrm{G} 3$ group were greater than those in the other groups. On day 0 after debonding, a large number of TGF-B1-positive osteoblast cells were detected on the surfaces of the alveolar bone. TGF- $\beta 1$ expression peaked in the G3 group 7 days after debonding, and the alterations were statistically significant. TGF- $\beta 1$ expression began to decrease on day 14 during relapse movement (-Table 1, - Fig. 3A). Compared with the expression observed on day 0 , the mean TGF- $\beta 1$ expression in the G3 group was increased by 1.926 positive cells/ field, while those of the G1 and G2 groups was increased by approximately 0.899 and 1.706 positive cells/field, respectively, on day 7 during relapse movement. As shown in

Table 1 Descriptive values and analysis of variance comparisons of transforming growth factor- $\beta 1$ and bone morphogenetic protein-2 expression (osteoblast-positive cells/field) and Runx-2 level (pg/mL)

\begin{tabular}{|c|c|c|c|c|c|}
\hline Parameter & \multirow[t]{2}{*}{ G1 } & \multirow[t]{2}{*}{ G2 } & \multirow[t]{2}{*}{ G3 } & \multirow[t]{2}{*}{$p$-Value } & \multirow[t]{2}{*}{ Post hoc comparison } \\
\hline TGF- $\beta 1$ expression & & & & & \\
\hline Day 0 & $9.177 \pm 1.963$ & $9.973 \pm 1.671$ & $11.036 \pm 2.023$ & $0.002^{\mathrm{a}}$ & $\mathrm{G} 1<\mathrm{G} 3$ \\
\hline Day 3 & $7.638 \pm 2.674$ & $10.234 \pm 1.041$ & $11.737 \pm 1.662$ & $0.004^{\mathrm{a}}$ & $\mathrm{G} 1<\mathrm{G} 2, \mathrm{G} 3$ \\
\hline Day 7 & $10.076 \pm 1.681$ & $11.679 \pm 1.195$ & $12.962 \pm 2.368$ & $0.004^{a}$ & $\mathrm{G} 1<\mathrm{G} 2<\mathrm{G} 3$ \\
\hline Day 14 & $8.433 \pm 2.375$ & $10.271 \pm 1.466$ & $11.532 \pm 1.035$ & 0.052 & NS \\
\hline Day 21 & $9.137 \pm 1.813$ & $10.173 \pm 1.367$ & $10.438 \pm 1.129$ & 0.136 & NS \\
\hline \multicolumn{6}{|l|}{ BMP-2 expression } \\
\hline Day 0 & $8.737 \pm 2.516$ & $9.039 \pm 1.671$ & $11.573 \pm 2.235$ & 0.282 & NS \\
\hline Day 3 & $6.733 \pm 1.244$ & $9.432 \pm 1.844$ & $11.967 \pm 1.062$ & $0.017^{a}$ & $\mathrm{G} 1<\mathrm{G} 2<\mathrm{G} 3$ \\
\hline Day 7 & $8.276 \pm 1.651$ & $11.176 \pm 2.054$ & $12.632 \pm 1.638$ & 0.091 & NS \\
\hline Day 14 & $11.033 \pm 2.027$ & $14.001 \pm 1.314$ & $16.774 \pm 2.459$ & $0.001^{\mathrm{a}}$ & $\mathrm{G} 1<\mathrm{G} 2<\mathrm{G} 3$ \\
\hline Day 21 & $8.771 \pm 2.431$ & $10.873 \pm 2.296$ & $14.173 \pm 1.179$ & $0.001^{\mathrm{a}}$ & $\mathrm{G} 1, \mathrm{G} 2<\mathrm{G} 3$ \\
\hline \multicolumn{6}{|l|}{ Runx-2 level } \\
\hline Day 0 & $66.677 \pm 8.321$ & $65.267 \pm 6.765$ & $66.971 \pm 5.237$ & 0.585 & NS \\
\hline Day 3 & $69.133 \pm 7.533$ & $70.113 \pm 6.126$ & $72.364 \pm 5.287$ & 0.332 & NS \\
\hline Day 7 & $42.136 \pm 8.089$ & $45.185 \pm 7.498$ & $45.989 \pm 8.211$ & 0.673 & NS \\
\hline Day 14 & $29.159 \pm 5.603$ & $32.729 \pm 7.082$ & $33.076 \pm 8.072$ & 0.098 & NS \\
\hline Day 21 & $21.729 \pm 4.043$ & $20.983 \pm 4.246$ & $23.183 \pm 5.239$ & 0.058 & NS \\
\hline
\end{tabular}

Abbreviations: ANOVA, analysis of variance; aPRF, autologous platelet-rich fibrin; BMP, bone morphogenetic protein; CHA, carbonate apatite; NS, not significant; TGF, transforming growth factor.

Note: Tested by one-way ANOVA and post hoc Tukey's HSD test; G1, the control group; G2, the CHA group; G3, the CHA-aPRF group. Values are presented as mean \pm standard deviation.

${ }^{a} p<0.05$, significant difference between groups. 
- Table 1, TGF- $\beta 1$ expression in the G3 group was significantly higher than that in the other groups on days 0,3 , and 7 after debonding $(p<0.05)$.

Immediately after the initiation of relapse movement, a few BMP-2-positive osteoblast cells were detected on the surface of the alveolar bone surface. BMP-2 expression peaked in all groups on day 14 , and the alterations were statistically significant ( $\mathbf{- T a b l e ~ 1 , ~ - ~ F i g . ~ 3 B ) . ~ C o m p a r e d ~ w i t h ~ t h e ~}$ expression observed on day 0 , the mean BMP-2 expression in the G3 group was increased by 5.201 positive cells/field, while those in the G1 and G2 groups was increased by approximately 2.296 and 4.962 positive cells/field, respectively, on day 14 during relapse movement. Quantitative evaluation revealed that the number of BMP-2-positive cells in the G3 group significantly increased on days 3, 14, and 21 compared with those in the other groups $(p<0.05)$.

Injection of CHA hydrogel-aPRF did not affect Runx-2 levels. ELISA indicated that although the Runx-2 level in the G3 group was slightly higher than those in the other groups, the differences noted among groups were not statistically significant ( $p>0.05$, - Table 1). As illustrated in

-Fig. 4, Runx-2 levels tended to decline after peaking on day 3 after appliance removal.

\section{Discussion}

This study confirms our hypothesis that CHA-aPRF injection could enhance osteoblastogenesis during orthodontic relapse. The result showed that $\mathrm{CHA}$ and $\mathrm{CHA}$ hydrogel-aPRF injection may increase TGF- $\beta 1$ and BMP-2 expression to higher levels during orthodontic relapse compared with those in the control group. The high microporosity of CHA allows antibiotics, growth factors, and antimicrobial agents to bind to its structure. ${ }^{17} \mathrm{CHA}$ hydrogel is designed as a drug delivery system on account of its ability to function as an intracellular protein transporter; the hydrogel can maintain the structure of proteins, such as the growth factors in aPRF, during delivery, and prevent their degradation or denaturation until they enters the intended area. ${ }^{13}$

Our results demonstrated that CHA-aPRF injection is effective in increasing TGF- $\beta 1$ expression, as it is indicated by the significant difference in number of TGF- $\beta 1$-positive cells between the CHA-aPRF injection, CHA injection, and control groups on days 0,3 , and 7 postdebonding. Concerning the mechanism, CHA appears to improve bone remodeling by enhancing the levels of phosphate and calcium, which are needed during new bone formation, in the surrounding tissue. ${ }^{18}$ When phosphate and calcium are released into the local environment, they stimulate osteoblastic proliferation. ${ }^{19}$ Osteoblast differentiation and proliferation is widely known to be regulated by TGF- $\beta 1$. TGF- $\beta 1$ plays many roles in enhancing osteoblast proliferation, including recruiting osteoblast precursors, or matrix-producing osteoblasts through chemotactic attraction and preventing osteoblast apoptosis..$^{20}$ The results showed that TGF- $\beta 1$ expression did not differ significantly among the three groups on days 14 and 21 postdebonding. TGF- $\beta 1$ expression is necessary to regulate the production of extracellular bone matrix protein in the early phase of osteoblast differentiation. ${ }^{21}$ In contrast to TGF- $\beta 1$, which inhibits the final stage of osteoblast differentiation and proliferation, BMP-2 promotes these activities. ${ }^{22}$

TGF- $\beta 1$ could reduce the ability of osteoblasts to secrete receptor activator of nuclear factor kappa- $\beta$ ligand (RANKL), an osteoclast differentiation factor, which means it can indirectly limit the formation and activation of osteoclasts and increase bone mass. ${ }^{23}$ TGF- $\beta 1$ is the strongest stimulator of bone formation that increases fibroblasts proliferation and stimulates the synthesis of collagen. ${ }^{24}$ TGF- $\beta 1$ signaling interruptions
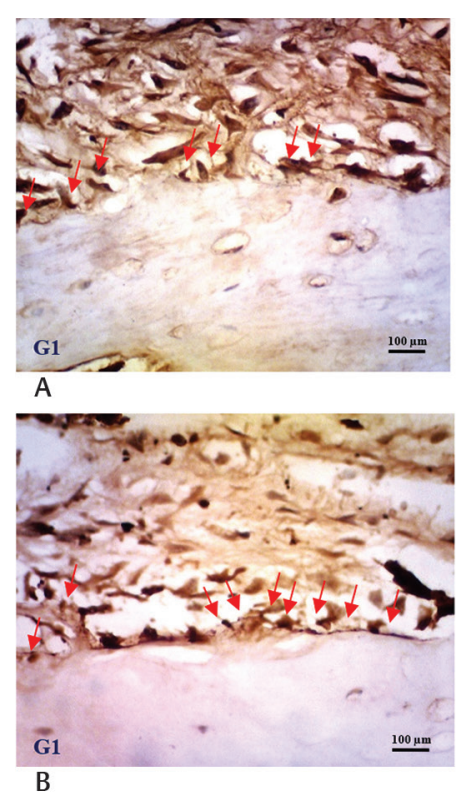
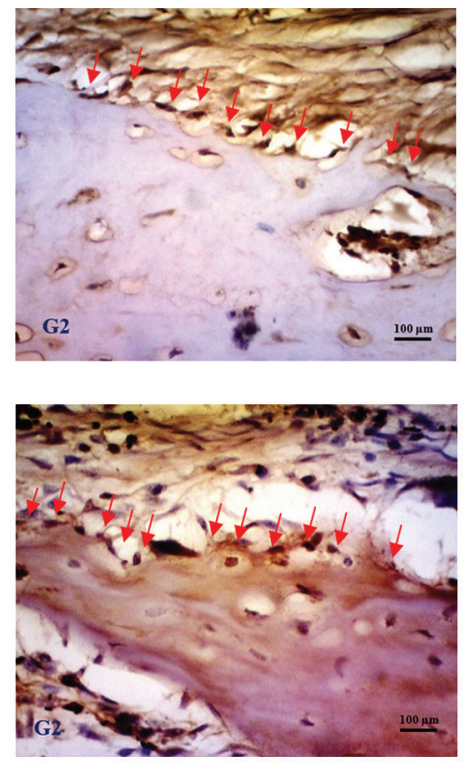
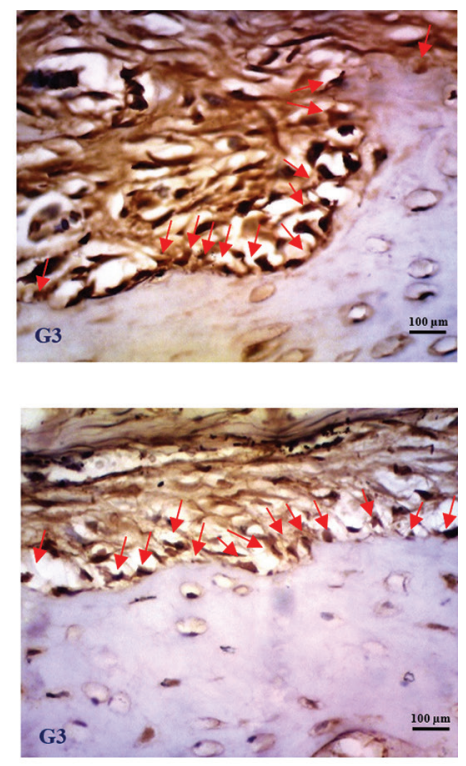

Fig. 3 Histological sections of experimental mandibles stained with immunohistochemical. During orthodontic relapse, G3 showed a greater number of transforming growth factor- $\beta 1$-positive cells (A) and bone morphogenetic protein-2-positive cells (B) compared with $\mathrm{G} 1$ and $\mathrm{G} 2$ group on day 7 after debonding. Scale bars represent $100 \mathrm{~mm}$ at $\times 400$ magnification. Red arrows represent osteoblast-positive cells. 


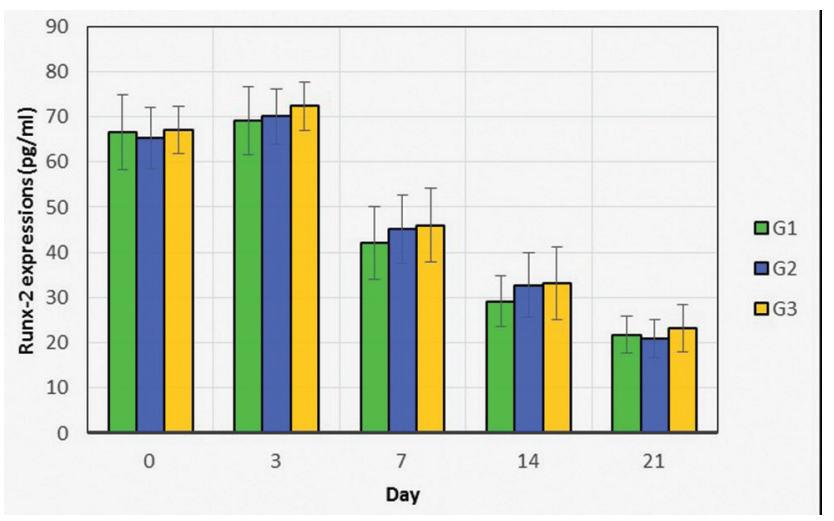

Fig. 4 Comparison of Runx-2 levels in the control (G1), CHA (G2), and CHA-aPRF (G3) groups from day 0 to 21 during relapse movement. The total Runx-2 level in G3 was only slightly higher than those in $\mathrm{G} 1$ and $\mathrm{G} 2$. The data are presented as mean \pm standard deviation. aPRF, autologous platelet-rich fibrin; CHA, carbonate apatite.

are known to cause extracellular matrix degeneration and decrease type 1 collagen expression in periodontal tissue, thereby triggering orthodontic relapse. ${ }^{25}$ These condition is expected in the process of orthodontic relapse prevention.

The results showed TGF- $\beta 1$ expression in the CHA-aPRF group is significantly higher than that in the CHA and control groups on days 0,3 , and 7 postdebonding. A possible mechanism behind the increase in TGF- $\beta 1$ expression involves the release of several growth factors, including IGF, TGF, vascular endothelial growth factor, and PDGF, from PRF. PRF performs a significant role in the enhanced release of some crucial growth factors such as TGF- $\beta 1 .{ }^{26}$ The aPRF contains higher concentrations of platelets and total growth factors than standard PRF. Modification of the aPRF preparation protocol induces a smaller number of cells to migrate to the bottom of the centrifuge tube so that the platelet and growth factor concentrations are more concentrated..$^{16}$ Growth factors are natural polypeptides that can stimulate the synthesis of the extracellular matrix and have the potential to induce osteoblastogenesis. ${ }^{27}$ Proper incorporation of CHA hydrogels with the growth factors contained in APRF is expected to be able to stimulate bone formation effectively. Growth factors are released gradually via a controlled-release system as the hydrogel is degraded and then induced osteogenesis through osteoconduction, osteoinduction, and angiogenesis. ${ }^{3}$

The results showed CHA-aPRF injection effectively increases BMP-2 expression. Autefage et $\mathrm{a}^{28}$ determined that CHA may increase BMP-2 expression, which is needed for bone regeneration. The osteoinductive ability of BMP-2 has been proven extensively in vitro and in vivo. BMP-2 is a TGF superfamily member that can increase the expression of osteocalcin, a noncollagen protein fraction present in the bone matrix that plays a vital role in bone remodeling, to induce new bone formation. ${ }^{24}$ Hassan et $\mathrm{al}^{29}$ stated that BMP-2 can induce new bone formation and improve periodontal tissue remodeling during OTM to prevent orthodontic relapse.
Our results showed that the expression of BMP-2 in the CHA-aPRF injection group is higher than that in the other groups on days 3,14 , and 21 postdebonding. Kizildağ et $\mathrm{a}^{30}$ determined that PRF may increase BMP-2 expression. PDGF in aPRF enhances osteoinduction by inducing osteoblastic cell differentiation and proliferation. ${ }^{27}$ It also acts as a master regulator of angiogenesis, which is necessary in bone remodeling to distribute the oxygen and nutrients needed by osteoprogenitor cells and provides a pathway for bone-forming precursor cells to reach the target area. ${ }^{1}$

Increased expression of BMP-2 can stimulate osteoblast maturation and induce alveolar bone formation to prevent relapse effectively. ${ }^{31}$ BMP-2 can reduce osteoclastogenesis activity through the RANKL-OPG pathway to increase bone mass. ${ }^{32}$ Osteoclastogenesis blocking has been shown to be effective in reducing relapse percentages after OTM. ${ }^{33}$ Our results demonstrated that BMP-2 expression peaks on day 14 postdebonding in the CHA-aPRF group. BMP-2 expression increases in the final stage of osteoblast differentiation..$^{22}$

Runx-2, a DNA-binding transcription factor that belongs to the Runt family, is essential for specifying osteogenic lineage. Runx-2 and Osterix are transcription factors that play a pivotal role in regulating early osteogenic differentiation from MSCs into osteoblasts. ${ }^{6,34}$ Runx-2 inhibits the differentiation of MSCs to several types of cells other than osteoblasts. Runx-2 is significant in uncommitted MSCs, and its expression is upregulated in preosteoblasts, highest in immature osteoblasts, and downregulated in mature osteoblasts. ${ }^{35}$ This phenomenon corresponds favorably with the results of our study, which revealed that Runx-2 levels tend to decrease after peaking on day 3. Our results also showed that Runx-2 levels do not significantly differ among the three groups tested. Levels of the activated Runx-2 protein in osteoblast precursors and osteoblasts must be assessed carefully. A previous study indicated that the continuous activation of Runx-2 in osteoblasts prevents osteoblast maturation, which favors the catabolic effect of parathyroid hormone. ${ }^{36}$ Runx-2 inhibits the maturation and transition of mature osteoblasts into osteocytes and maintains them in a resting state. ${ }^{21}$ Additionally, Runx-2 controls RANKL and OPG expression by stimulating osteoclast differentiation. ${ }^{37}$ Runx-2 is involved in osteoclastogenesis through the induction of Tnfsf11 expression and inhibition of Tnfrsf11b expression. ${ }^{36}$ However, the molecular mechanisms through which Runx-2 interferes with improvements in osteoclastogenesis must be further investigated.

Taken together, the results of this preclinical study suggest that the induction of osteoblastogenesis mediated by CHA hydrogel-aPRF is a potential target to minimize orthodontic relapse. The combination of CHA hydrogel and aPRF resulted in synergistic effects that may stimulate complex molecular events and interactions leading to osteoblastogenesis. However, due to the limitations of this study, including the lack of aPRF stock, which should be used immediately after preparation, the translation of these results to the clinical setting requires further study. Our future work will focus on developing a suitable formulation material for orthodontic clinical use. For example, the freeze-dried form of aPRF for material long-term stability. 
In addition, the dose of materials used in animal studies must be modified when applied to humans, and additional randomized, controlled clinical trials are needed to test the ultimate outcomes associated with the materials.

\section{Conclusion}

Although intrasulcular injection of hydrogel-CHA incorporated aPRF can positively upregulate TGF- $\beta 1$ and BMP-2 expression, it cannot enhance Runx-2 levels during orthodontic relapse in rabbits.

\section{Funding}

This work was supported by Universitas Gadjah Mada, Indonesia.

\section{Conflict of Interest}

None declared.

\section{References}

1 Alhasyimi AA, Pudyani PP, Asmara W, Ana ID. Enhancement of post-orthodontic tooth stability by carbonated hydroxyapatite-incorporated advanced platelet-rich fibrin in rabbits. Orthod Craniofac Res 2018;21(2):112-118

2 Kaan M, Madléna M. [Retention and relapse. Review of the literature]. Fogorv Sz 2011;104(4):139-146

3 Alhasyimi AA, Pudyani PS, Asmara W, Ana ID. Locally inhibition of orthodontic relapse by injection of carbonated hydroxy apatite-advanced platelet rich fibrin in a rabbit model. Key Eng Mater 2017;758:255-263

4 Nanjundaiah SM, Astry B, Moudgil KD. Mediators of inflammation-induced bone damage in arthritis and their control by herbal products. Evid Based Complement Alternat Med 2013;2013:518094

5 Wu M, Chen G, Li YP. TGF-ßand BMP signaling in osteoblast, skeletal development, and bone formation, homeostasis and disease. Bone Res 2016;4(4):16009

6 Nugraha AP, Narmada IB, Ernawati DS, et al. Osteogenic potential of gingival stromal progenitor cells cultured in platelet rich fibrin is predicted by core-binding factor subunit- $\alpha 1$ / Sox9 expression ratio (in vitro). F1000 Res 2018;7:1134

7 Kartal Y, Kaya B. Fixed orthodontic retainers: a review. Turk J Orthod 2019;32(2):110-114

$8 \mathrm{Al}$ Yami EA, Kuijpers-Jagtman AM, van't Hof MA. Stability of orthodontic treatment outcome: follow-up until 10 years postretention. Am J Orthod Dentofacial Orthop 1999;115(3):300-304

9 Alhasyimi AA, Rosyida NF, Rihadini MS. Postorthodontic relapse prevention by administration of grape seed (vitis vinifera) extract containing cyanidine in rats. Eur J Dent 2019;13(4):629-634

10 Nugraha AP, Rezkita F, Putra KG, Narmada IB, Ernawati DS, Rantam FA. Triad tissue engineering: gingival mesenchymal stem cells, platelet rich fibrin and hydroxyapatite scaffold to ameliorate relapse post orthodontic treatment. Biochem Cell Arch 2019;19(2):3689-3693

11 Fujioka-Kobayashi M, Tsuru K, Nagai H, et al. Fabrication and evaluation of carbonate apatite-coated calcium carbonate bone substitutes for bone tissue engineering. J Tissue Eng Regen Med 2018;12(10):2077-2087
12 Alhasyimi AA, Pudyani PS, Asmara W, Ana ID. Effect of carbonated hydroxyapatite incorporated advanced platelet-rich fibrin intramuscular injection on the alkaline phosphatase level during orthodontic relapse. AIP Conf Proc 2018;1933(1):030006

13 Tada S, Chowdhury EH, Cho CS, Akaike T. pH-sensitive carbonate apatite as an intracellular protein transporter. Biomaterials 2010;31(6):1453-1459

14 Dohle E, El Bagdadi K, Sader R, Choukroun J, James Kirkpatrick C, Ghanaati S. Platelet-rich fibrin-based matrices to improve angiogenesis in an in vitro co-culture model for bone tissue engineering. J Tissue Eng Regen Med 2018;12(3):598-610

15 Chang IC, Tsai CH, Chang YC. Platelet-rich fibrin modulates the expression of extracellular signal-regulated protein kinase and osteoprotegerin in human osteoblasts. J Biomed Mater Res A 2010;95(1):327-332

16 Ghanaati S, Booms P, Orlowska A, et al. Advanced platelet-rich fibrin: a new concept for cell-based tissue engineering by means of inflammatory cells. J Oral Implantol 2014;40(6):679-689

17 Sivakumar M, Panduranga Rao K. Preparation, characterization and in vitro release of gentamicin from coralline hydroxyapatite-gelatin composite microspheres. Biomaterials 2002;23(15):3175-3181

18 Ana ID, Matsuya S, Ishikawa K. Engineering of carbonate-apatite bone substitute based on composition-transformation of gypsum and calcium-hydroxide. Engineering (Lond) 2010;2:344-352

$19 \mathrm{Wu} \mathrm{X}$, Itoh $\mathrm{N}$, Taniguchi $\mathrm{T}$, Nakanishi T, Tanaka K. Requirement of calcium and phosphate ions in expression of sodium-dependent vitamin $C$ transporter 2 and osteopontin in MC3T3-E1 osteoblastic cells. Biochim Biophys Acta 2003;1641(1):65-70

20 Kasagi S, Chen W. TGF-beta1 on osteoimmunology and the bone component cells. Cell Biosci 2013;3(1):4

21 Alliston T, Choy L, Ducy P, Karsenty G, Derynck R. TGF-beta-induced repression of CBFA1 by Smad3 decreases cbfa 1 and osteocalcin expression and inhibits osteoblast differentiation. EMBO J 2001;20(9):2254-2272

22 Canalis E, Economides AN, Gazzerro E. Bone morphogenetic proteins, their antagonists, and the skeleton. Endocr Rev 2003;24(2):218-235

23 Chen G, Deng C, Li YP. TGF-and BMP signaling in osteoblast differentiation and bone formation. Int $\mathrm{J}$ Biol Sci 2012;8(2):272-288

24 Narmada IB, Rubianto M, Putra ST. The role of low-intensity biostimulation laser therapy in transforming growth factor1, bone alkaline phosphatase and osteocalcin expression during orthodontic tooth movement in cavia porcellus. Eur J Dent 2019;13(1):102-107

25 Feng L, Yang R, Liu D, et al. PDL progenitor-mediated PDL recovery contributes to orthodontic relapse. J Dent Res 2016;95(9):1049-1056

26 Tehranchi A, Behnia H, Pourdanesh F, Behnia P, Pinto N, Younessian F. The effect of autologous leukocyte platelet rich fibrin on the rate of orthodontic tooth movement: a prospective randomized clinical trial. Eur J Dent 2018;12(3):350-357

27 Galav S, Chandrashekar KT, Mishra R, Tripathi V, Agarwal R, Galav A. Comparative evaluation of platelet-rich fibrin and autogenous bone graft for the treatment of infrabony defects in chronic periodontitis: clinical, radiological, and surgical reentry. Indian J Dent Res 2016;27(5):502-507

28 Autefage H, Briand-Mésange F, Cazalbou S, et al. Adsorption and release of BMP-2 on nanocrystalline apatite-coated and uncoated hydroxyapatite/beta-tricalcium 
phosphate porous ceramics. J Biomed Mater Res B Appl Biomater 2009;91(2):706-715

29 Hassan AH, Al-Hubail A, Al-Fraidi AA. Bone inductive proteins to enhance postorthodontic stability. Angle Orthod 2010;80(6):1051-1060

30 Kizildağ A, Çiçek Y, Arabaci T, Köse O. The effect of leukocyte-platelet-rich fibrin on bone morphogenetic protein-2 and insulin-like growth factor-1 levels in patients with chronic periodontitis: a randomized split mouth clinical trial. Growth Factors 2019;36(6):1-7

31 Chen YP, Han GH, Jin CW, Shi RX, Hou JH. [Effect of simvastatin on bone morphogenetic protein-2 expression in the periodontal tissue after rat tooth movement]. Zhonghua Kou Qiang Yi Xue Za Zhi 2008;43(1):21-25

32 Kamiya N, Ye L, Kobayashi T, et al. Disruption of BMP signaling in osteoblasts through type IA receptor (BMPRIA) increases bone mass. J Bone Miner Res 2008;23(12):2007-2017
33 Hudson JB, Hatch N, Hayami T, et al. Local delivery of recombinant osteoprotegerin enhances postorthodontic tooth stability. Calcif Tissue Int 2012;90(4):330-342

34 Nugraha AP, Narmada IB, Ernawaty DS, et al. The aggrecan expression post platelet rich fibrin administration in gingival medicinal signaling cells in Wistar rats (Rattus novergicus) during the early osteogenic differentiation (in vitro) Kafkas Univ Vet Fak Derg 2019;25(3):421-425

35 Komori T. Regulation of proliferation, differentiation and functions of osteoblasts by Runx-2. Int J Mol Sci 2019;20(7):1-11

36 Komori T. Regulation of bone development and maintenance by Runx2. Front Biosci 2008;13:898-903

37 Enomoto $\mathrm{H}$, Shiojiri S, Hoshi K, et al. Induction of osteoclast differentiation by Runx2 through receptor activator of nuclear factor-kappa B ligand (RANKL) and osteoprotegerin regulation and partial rescue of osteoclastogenesis in Runx2-/- mice by RANKL transgene. J Biol Chem 2003;278(26):23971-23977 